\title{
Circular RNA atlas in osteoclast differentiation with and without alendronate treatment
}

Jianbiao Lin ${ }^{1 \dagger}$, Shaofeng $\mathrm{Ma}^{2 \dagger}$, Cong Zhu ${ }^{1 \dagger}$, Changqing Chen ${ }^{1}$, Weibin Lin ${ }^{1}$, Canbin Lin ${ }^{1}$, Guofeng Huang ${ }^{1 *}$ and Zhenqi Ding ${ }^{1 *}$ (D)

\begin{abstract}
Background: Alendronate (AL) is the most widely used bisphosphonate in the treatment of osteoporosis (OP). However, the role of circular RNAs (circRNAs) in the treatment of OP with AL remains unclear.

Methods: In this study, we showed that osteoclast (OC) precursors (OPCSs) could be induced into OCs with macrophage colony-stimulating factor (MCSF) and receptor activator of nuclear factor-KB ligand (RANKL) treatment. Subsequently, the OCs were treated with AL. OC differentiation-related biomarkers including RANK, tartrate-resistant acid phosphatase (TRAP), and cathepsin K (CTSK) were analyzed with TRAP staining, quantitative real-time (qPCR), and western blotting. Differentially expressed circRNAs (DECs) were identified among the OPCS, OC, and OC $+\mathrm{AL}$ groups. In addition, the expression levels of 10 DECs related to OC differentiation were verified by qPCR.

Results: TRAP staining showed that MCSF and RANKL treatment effectively induced OPCSs to differentiate into OCs. In addition, GPCR and western blot analysis revealed that the three biomarkers of OC (RANK, TRAP, and CTSK) were expressed significantly more in the OC group than those in the OPCS group. In contrast, the mRNA and protein expression levels of these three biomarkers decreased significantly in OCs treated with AL compared with those non-treated OCs. GO analysis of the DECs in the OPCS group vs. the OC group revealed that their functions were mainly related to cell, cell part, binding, and single-organism terms. KEGG analysis of the top 20 DECs in a comparison between the OPCS and OC groups showed that genes involved in mitogen-activated protein kinase signaling were the most common. Results of functional analyses of DECs in an OC vs. OC + AL comparison were similar to those in the OPCS vs. OC comparison. Finally, qPCR showed that, in the OC + AL vs. OC group comparison, the expression levels of seven and three DECs significantly decreased and increased, respectively.

Conclusions: Having successfully induced OPCSs to differentiate into OCs, we showed that AL suppresses the differentiation of OPCS into OC and that 10 DECs were involved in the regulation of this process. This indicates that these DECs might be important to the treatment of OP.
\end{abstract}

Keywords: Alendronate, Osteoporosis, MCSF, RANKL

\footnotetext{
*Correspondence: huangquofeng@xmu.edu.cn; dingzhenqi175@163.com

${ }^{\dagger}$ Jianbiao Lin, Shaofeng Ma and Cong Zhu contributed equally to this work.

'Center for Orthopedics, Affiliated Southeast Hospital of Xiamen University/

909th Hospital of People's Liberation Army, 269 Zhanghua Middle Road,

Zhangzhou 363000, Fujian, China

Full list of author information is available at the end of the article
}

(C) The Author(s). 2020 Open Access This article is licensed under a Creative Commons Attribution 4.0 International License, which permits use, sharing, adaptation, distribution and reproduction in any medium or format, as long as you give appropriate credit to the original author(s) and the source, provide a link to the Creative Commons licence, and indicate if changes were made. The images or other third party material in this article are included in the article's Creative Commons licence, unless indicated otherwise in a credit line to the material. If material is not included in the article's Creative Commons licence and your intended use is not permitted by statutory regulation or exceeds the permitted use, you will need to obtain permission directly from the copyright holder. To view a copy of this licence, visit http://creativecommons.org/licenses/by/4.0/ The Creative Commons Public Domain Dedication waiver (http://creativecommons.org/publicdomain/zero/1.0/) applies to the data made available in this article, unless otherwise stated in a credit line to the data. 


\section{Introduction}

Osteoporosis (OP) is among the most common bone diseases worldwide. It is characterized by a reduction in bone tissue volume and bone density per unit volume, and it is caused by the loss of bone calcium and bone matrix [1]. Because the average age of populations is generally increasing, OP is becoming a worldwide health problem. Indeed, according to the International Osteoporosis Foundation, more than 200 million people worldwide have OP, with the most common type being postmenopausal OP [2] (which about 30\% of postmenopausal women suffer from). The most important mechanisms of postmenopausal OP are the excessive activation of osteoclasts (OCs) and the inhibition of osteoblasts caused by estrogen deficiency. Moreover, attenuation of osteoblast formation and/or increased bone resorption by OCs is a key mechanism of pathogenesis in OP; thus, OC dysfunction is an important OP pathogenic factor $[3,4]$. Although previous studies have revealed multiple molecular mechanisms for OC dysfunction, few have investigated the roles of circular RNAs (circRNAs) during drug-related regulation of OC differentiation.

Osteoblasts and OCs are the main functional cells of bone formation: osteoblasts are responsible for new bone formation, whereas OCs are responsible for aged bone resorption [5]. Thus, these cells maintain a dynamic balance of formation and resorption; however, when this balance is disrupted, the function or architecture of the bone becomes abnormal [6]. Consequently, drugs for the treatment of OP mainly include bone resorption inhibitors and bone formation promoters. One such drug is alendronate $(\mathrm{AL})$, which is a bisphosphonate widely used for OP treatment. AL has multiple effects in OP patients including inhibiting $\mathrm{OC}$ activity, increasing bone density, and reducing the incidence of vertebral and nonvertebral fractures $[7,8]$. Previous studies have suggested that bisphosphonate's mechanism of action is dependent on three processes: (1) altering the morphology and structure of the OCs to inhibit their function; (2) inhibiting the production of osteoblast-mediated interleukin (IL) -6 and tumor necrosis factor- $\alpha$, thereby decreasing OC genesis; and (3) physically and chemically bonding with the bone matrix, thereby interfering with bone resorption [9]. However, the role of circRNAs in the underlying molecular mechanisms of AL's effects in OP remains to be elucidated.

circRNAs are covalently closed RNA molecules that are generated through a process named back-splicing [10] and are involved in the regulation of various biological processes. For example, circRNA.014511 inhibits P53 expression by binding to miR-29b-2-5p, and it reduces the sensitivity of bone marrow mesenchymal stem cells by regulating cell apoptosis and the cell cycle [11]. Additionally, circRNA.33186-knockdown inhibits apoptosis and promotes proliferation in chondrocytes treated with IL-1 $\beta$ [12]. Also, circRNA.28313 functions in macrophage colony-stimulating factor (MCSF) + receptor activator of nuclear factor- $\mathrm{k} B$ ligand (RANKL)-induced OC differentiation via the regulation of miR-195a expression; this affects bone absorption in mice [13]. Moreover, at different stages of mouse OC growth, the expression levels of various circRNAs are different [14].

Unfortunately, OCs are difficult to separate from cortical bone in vitro: they are terminally differentiated cells lacking proliferation abilities [15]. Therefore, OCs cannot be isolated for culture in vitro. However, THP-1 cells can differentiate into OCs when stimulated by phorbol-12 myristate-13 acetate (PMA), RANKL, and MCSF $[16,17]$. Thus, to investigate the involvement of circRNAs in the AL treatment of OP, THP-1 cells were used in the present study to differentiate into OCs for further functional analysis. Specifically, we probed the potential circRNA atlas involved in AL-induced OC differentiation. From our results, we were able to draw a circRNA-miRNA-mRNA network of AL-induced OC differentiation. Our results provide insights that could be used for further clinical studies of OP treatment.

\section{Materials and methods \\ Cell treatment}

THP-1 cells were purchased from ATCC (VA, USA) and maintained in RPMI 1640 with 10\% fetal bovine serum (Invitrogen, Carlsbad, CA, USA) and 1\% penicillin/ streptomycin (HyClone, USA). Cell lines were maintained in a humidified chamber at $5 \% \mathrm{CO}_{2}$ and $37^{\circ} \mathrm{C}$. THP-1 cells were induced into OC precursors (OPCSs) through stimulation with $100 \mathrm{ng} / \mathrm{mL}$ PMA for 3 days. The detailed experimental procedure for this process was previously described by Takashiba et al. [18]. Once OPCSs were available, they were uniformly inoculated into 96-well plates (5000 cells/well); they were induced over 7 days into OCs using a final concentration that contained $100 \mathrm{ng} / \mathrm{mL}$ RANKL and $50 \mathrm{ng} / \mathrm{mL}$ MCSF. Every 72 $\mathrm{h}$ during induction, the culture medium was changed and the state of OC differentiation was evaluated. During OC differentiation, $10^{-8} \mathrm{M}$ AL was either added or not added (depending on the treatment group) to the induction culture on day 4. After 7 days, the treated cells were harvested and divided into three groups: the OPCS group, $\mathrm{OC}$ group, and $\mathrm{OC}+\mathrm{AL}$ group.

\section{Tartrate-resistant acid phosphatase (TRAP) staining}

Here, TRAP staining was performed by modifying the method of Oshima et al. [19]. Briefly, OCs were incubated for $15 \mathrm{~min}$ at $37^{\circ} \mathrm{C}$ in freshly prepared $0.1 \mathrm{M}$ Tris buffer (pH 5.0) that contained $1.35 \mathrm{mM}$ naphthol ASMX phosphate (Sigma); $0.362 \mathrm{MN}, \mathrm{N}$-dimethylformamide; $3.88 \mathrm{mM}$ Violet LB salt (Sigma); and $25 \mathrm{mM}$ 
Table 1 Primer sequences of circRNAs

\begin{tabular}{|c|c|c|c|}
\hline CircBase ID & Sequence (5' to $\left.3^{\prime}\right)$ & Product length & Gene ID \\
\hline \multirow[t]{2}{*}{ hsa_circ_0000284 } & TCTCGGTACTACAGGTATGGC & 193 & HIPK3 \\
\hline & ACCCTTAGTGGGAGGATGAGA & & \\
\hline \multirow[t]{2}{*}{ hsa_circ_0000638 } & CGGGAAAAATAGTAGCACCAGC & 173 & ETFA \\
\hline & TGCCACCTTGTCACATTTGG & & \\
\hline \multirow[t]{2}{*}{ hsa_circ_0000994 } & TGAAATTGTTAGGTTGTGACAGTTG & 158 & SLC8A1 \\
\hline & TCTCCTTCCATTTCTGTCTCAGC & & \\
\hline \multirow[t]{2}{*}{ hsa_circ_0001776 } & CAAGGAACCTTCCGGGTGTT & 143 & ESYT2 \\
\hline & GCTTTGGAAGATTTGGTTGCCA & & \\
\hline \multirow[t]{2}{*}{ hsa_circ_0002922 } & TGAGGCAAGACTTCTAACTCGG & 165 & ZNF124 \\
\hline & GCTCTGGTCTTCCCCTTTGT & & \\
\hline \multirow[t]{2}{*}{ hsa_circ_0003249 } & AATCATTCCTGTGATGCCAGA & 186 & LRP11 \\
\hline & TGAGGCACCTTCATGTCCAC & & \\
\hline \multirow[t]{2}{*}{ hsa_circ_0007710 } & CCAGAAGGAAGACAATGCTGTT & 173 & ELF2 \\
\hline & AGTCAATAGAGATGGAGTGGAGT & & \\
\hline \multirow[t]{2}{*}{ hsa_circ_0094798 } & ATGAGAATGGCATCTAAGAATGAAG & 163 & CWF19L2 \\
\hline & CCCTTTCTCTITCCTCATGGGT & & \\
\hline \multirow[t]{2}{*}{ hsa_circ_0101874 } & GGACAGCCTGATGCCAAAAC & 200 & FKBP3 \\
\hline & CAGACTTGGTTTCTTGGGTTTA & & \\
\hline \multirow[t]{2}{*}{ hsa_circ_0113954 } & ACAAAGAAAATGAAAAAGGAAGGCG & 173 & MIER1 \\
\hline & TCCCCACTACAGCCACTGTT & & \\
\hline
\end{tabular}

Table 2 Primer sequences of $\mathrm{qPCR}$

\begin{tabular}{|c|c|c|c|}
\hline Gene & Primer & Sequence (5' to $3^{\prime}$ ) & Product length (bp) \\
\hline \multirow[t]{2}{*}{ HIPK3 } & H-HIPK3-F & CAGTCTTCCTTCTCCGCTCC & 166 \\
\hline & H-HIPK3-F & CTTCCTTCCCGGGGATTTGG & \\
\hline \multirow[t]{2}{*}{ ETFA } & H-ETFA-F & CTCCACCAGCCTAATCAACCT & 196 \\
\hline & H-ETFA-F & TGGAGCTGGGTCAGGTTTAAG & \\
\hline \multirow[t]{2}{*}{ SLC8A1 } & H-SLC8A1-F & CATCGAAGGGACTGCCAGAG & 181 \\
\hline & $\mathrm{H}-\mathrm{SLC} 8 \mathrm{~A} 1-\mathrm{F}$ & CTCACTCATCTCCACCAGGC & \\
\hline \multirow[t]{2}{*}{ ESYT2 } & H-ESYT2-F & AAGGAACCTTCCGTCAGGG & 120 \\
\hline & H-ESYT2-F & TCCCACACAGGTTCATTGGT & \\
\hline \multirow[t]{2}{*}{ ZNF124 } & H-ZNF124-F & CTTCTCCTTCACTCTCGGCG & 180 \\
\hline & H-ZNF124-F & TCCAACAAAGCCCACTCCTC & \\
\hline \multirow[t]{2}{*}{ LRP11 } & H-LRP11-F & TGAGTCAAAGGGTGATGGAGG & 121 \\
\hline & H-LRP11-F & TAGTCGGCATGCAACCATGA & \\
\hline \multirow[t]{2}{*}{ ELF2 } & H-ELF2-F & TCCTTCTCTCCCTGTGGAGC & 151 \\
\hline & $\mathrm{H}$-ELF2-F & TCTITGGAACTGCTCTCCGC & \\
\hline \multirow[t]{2}{*}{ CWF19L2 } & H-CWF19L2-F & TGGTGGATTAAGCTGGCTAAGG & 195 \\
\hline & H-CWF19L2-F & TATGTGGGTTTCCTCCACCG & \\
\hline \multirow[t]{2}{*}{ FKBP3 } & $\mathrm{H}-\mathrm{FKBP3-F}$ & AGTAAAGCGGAGGCAGCG & 191 \\
\hline & $\mathrm{H}-\mathrm{FKBP3-F}$ & TGTTAGCTGTCTTGGCCACAT & \\
\hline \multirow[t]{2}{*}{ MIER1 } & H-MIER1-F & TGAATCTTCAAGTCCAGGAGGT & 182 \\
\hline & H-MIER1-F & GAATTGGCATGTCGCCTTCC & \\
\hline
\end{tabular}




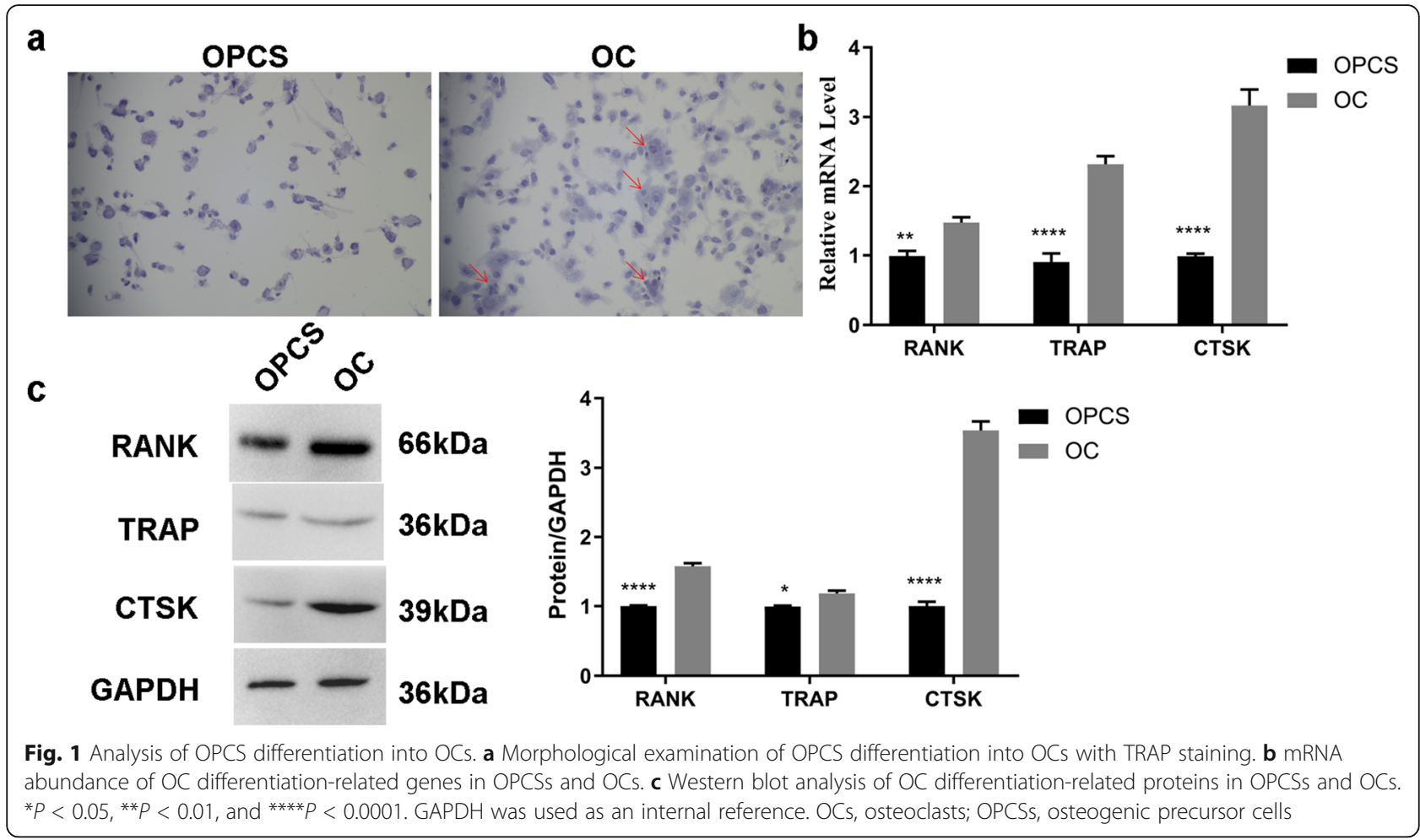

sodium tartrate. Slides were rinsed for $10 \mathrm{~min}$ and counterstained with hematoxylin. One-micromolar sections were cut using a Sorvall Porter-blum MT-2B ultra microtome. New bone formation was visualized using fluorescence microscopy (Nikon, Tokyo, Japan).

\section{Real-time quantitative PCR (qPCR)}

Reverse transcription of mRNA from the three treatment groups was carried out using a final volume of $100 \mu \mathrm{L}$ from $400 \mathrm{ng}$ total RNA and with a High-capacity cDNA Archive kit (Applied Biosystems) according to the manufacturer's instructions. mRNA levels were determined by $\mathrm{qPCR}$, and the following primers were used: RANK forward 5'-TCTGCTTCTCTTCGCGTCTG-3';
RANK reverse 5' ${ }^{\prime}$-AGCCTCATTGATCCAGTGCC-3'; TRAP forward 5'-GATCCCACAGACCAATGTGTC-3'; TRAP reverse 5'-CCAGCACGTAGTCCTCCCT-3'; cathepsin $\mathrm{K}$ (CTSK) forward $5^{\prime}$-TCCTGTTGGGCTTT TAGCTC-3'; CTSK reverse 5'-GTCATGTAGCCCCC TCCAC-3. Reactions were performed in $50-\mu \mathrm{L}$ volumes containing SYBR Green PCR master mix (PerkinElmer Biosystems). qPCR was performed using 96well optical plates in a GeneAmp PCR System 9600 (Perkin-Elmer Biosystems). Thermal cycling conditions were as follows: $2 \mathrm{~min}$ at $50^{\circ} \mathrm{C}$ and $10 \mathrm{~min}$ at $95^{\circ} \mathrm{C}$, followed by 40 cycles at $95^{\circ} \mathrm{C}$ for $30 \mathrm{~s}, 60^{\circ} \mathrm{C}$ for $30 \mathrm{~s}$, and $72{ }^{\circ} \mathrm{C}$ for $2 \mathrm{~min}$. Data were collected using an ABI analytical thermal cycler. mRNA

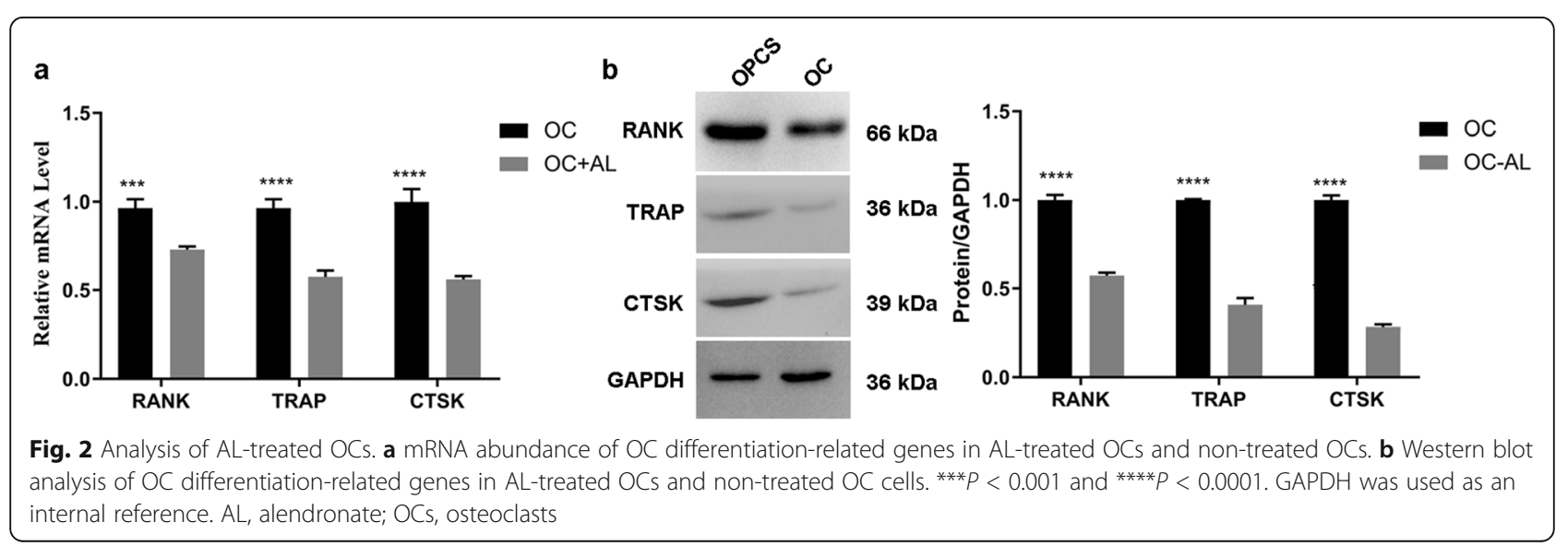


expression levels were calculated on the basis of a relative standard curve and using the $2^{-\Delta \Delta \text { ct }}$ method. In addition, we designed 20 primer pairs to identify the linear DNA and corresponding circRNAs; the primer sequences for these are listed in Tables 1 and 2 . PCR products were analyzed using agarose gel electrophoresis (1.5\%).

\section{Western blot analysis}

Total cellular protein from the three treatment groups was isolated by the addition of $1 \%$ PMSF and RIPA lysis buffer (containing $50 \mathrm{mM}$ Tris- $\mathrm{HCl}$ at $\mathrm{pH} 7.4,150 \mathrm{mM} \mathrm{NaCl}, 1 \%$
NP-40, and 0.1\% SDS). After boiling with SDS-PAGE sample buffer for $5 \mathrm{~min}$, the samples were subjected to SDSPAGE. The proteins were then transferred to a PVDF membrane (Millipore, USA). After blocking for $1 \mathrm{~h}$ at room temperature, the membrane was incubated with a 1:1000 dilution of rabbit polyclonal anti-mouse RANK, TRAP, CTSK, or GAPDH antibodies (ABGENT, USA) overnight. Before detection with an enhanced chemiluminescence detection kit (Advansta, USA), proteins were incubated with the corresponding secondary antibody for $1 \mathrm{~h}$ at room temperature. The bands were obtained by GeneGnome 5 (Synoptics Ltd., UK).

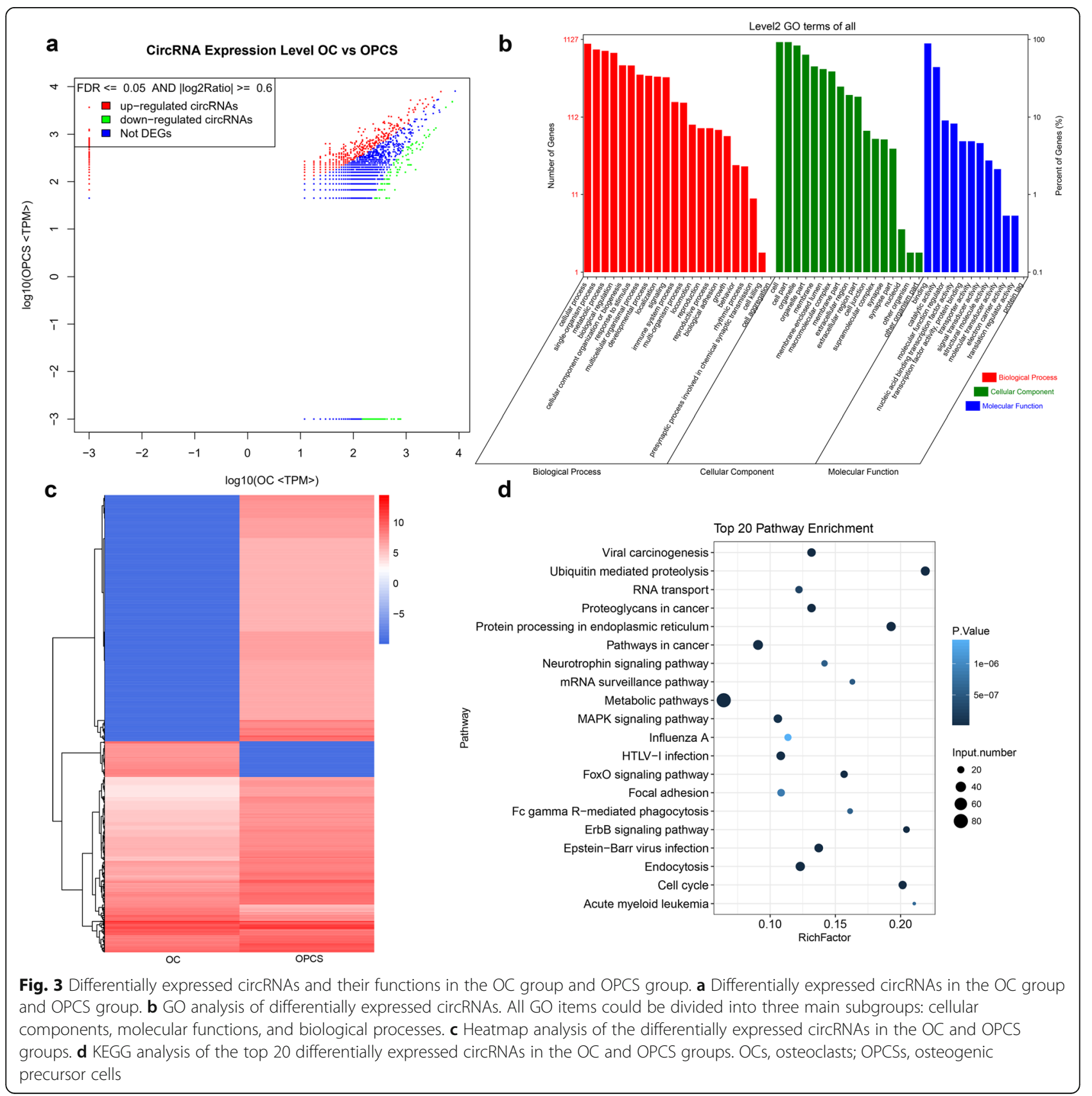




\section{Bioinformatics}

Differentially expressed circRNAs (DECs) were determined using the following parameters: $P$ value $\leq 0.05$ and $\mid \log _{2}$ Ratio $\mid \geq 2$. GO enrichment analysis and KEGG analysis of DECs were carried out using the online database KOBAS3.0 (http://kobas.cbi.pku.edu.cn/) with the default parameters maintained. In addition, a hypergeometric test was used to calculate the major biochemical metabolic pathways in which the DECs were involved.

\section{Data analysis}

SPSS V16.0 software (IBM, USA) was used for statistical analyses. Statistical differences between groups were calculated using Tukey's test. Where $P$ was $<0.05$, this indicated a significant difference.

\section{Results}

\section{OC differentiation and drug induction}

In order to investigate the OC differentiation and AL induction of OCs, THP-1 cells were induced into OPCSs by PMA treatment. Subsequently, OPCSs were further induced into OCs by MCSF and RANKL treatment. Finally, AL was used to treat OC. TRAP staining showed that MCSF and RANKL treatment effectively induced OPCS to differentiate into OCs (Fig. 1a). Moreover, qPCR showed that the expression levels of the OC differentiation-related biomarkers RANK, TRAP, and CTSK were significantly increased in OCs compared with OPCSs $(P<0.05)$ (Fig. 1b). Western blot analysis confirmed these results $(P<0.05)$ (Fig. 1c). In the AL induction of OCs, qPCR showed that the expression levels of RANK, TRAP, and CTSK were significantly decreased in AL-treated OCs compared with those in untreated OCs $(P$ $<0.05$ ) (Fig. 2a). Similar results were observed in western blot analysis (Fig. 2b). Thus, AL apparently inhibited the differentiation of OCs.

\section{DECs in an OPCS group vs. OC group comparison}

We identified the circRNAs that were differentially expressed in the OPCS and OC groups. Figure 3a shows that the number of upregulated DECs (1394) was higher than the number of downregulated DECs (214) in the OPCS group vs. the OC group. GO analysis revealed that the functions of these DECs were mainly distributed into three categories: cellular component, molecular function, and biological process (Fig. 3b). In the cellular component category, "cell," "cell part," and "organelle" were the three most enriched items. In the molecular function category, "binding" was the most enriched item. Finally, in the biological process category, "cellular process" and "single-organism process" were the most enriched items. We also produced a heat map to show the cluster relationships among these DECs (Fig. 3c). KEGG analysis of the top 20 DECs showed that enrichment was mostly in metabolic pathways and in the mitogen-activated protein kinase (MAPK) signaling pathway (Fig. 3d and Table 3).

Table 3 Top 20 differential expression circRNA list between the OC group and OPCS group

\begin{tabular}{|c|c|c|c|c|c|c|c|}
\hline CircBase_ID & Gene ID & OC-TPM & OPCS-TPM & $\log _{2}$ ratio $(\mathrm{OPCS} / \mathrm{OC})$ & Up-downregulation & $\boldsymbol{P}$ value & FDR \\
\hline hsa_circ_0003307 & TALDO1 & 11.919 & 269.645 & 4.5 & Up & $2.05 \mathrm{E}-07$ & $1.77 \mathrm{E}-05$ \\
\hline hsa_circ_0007761 & ATXN7 & 29.796 & 629.171 & 4.4 & Up & $3.76 \mathrm{E}-15$ & $1.14 \mathrm{E}-12$ \\
\hline hsa_circ_0076742 & MCM3 & 35.756 & 629.171 & 4.137 & Up & $1.75 E-14$ & $5.21 \mathrm{E}-12$ \\
\hline hsa_circ_0072202 & C5orf42 & 11.919 & 202.234 & 4.085 & Up & $1.42 \mathrm{E}-05$ & 0.000626866 \\
\hline hsa_circ_0040123 & PDXDC2P & 17.878 & 269.645 & 3.915 & Up & $8.83 \mathrm{E}-07$ & $6.07 \mathrm{E}-05$ \\
\hline hsa_circ_0003260 & RAP1B & 17.878 & 269.645 & 3.915 & Up & $8.83 \mathrm{E}-07$ & $6.05 \mathrm{E}-05$ \\
\hline hsa_circ_0030213 & $\mathrm{LRCH} 1$ & 11.919 & 179.763 & 3.915 & Up & $5.66 \mathrm{E}-05$ & 0.001799064 \\
\hline hsa_circ_0017065 & B3GALNT2 & 11.919 & 179.763 & 3.915 & Up & $5.66 \mathrm{E}-05$ & 0.001795578 \\
\hline hsa_circ_0008842 & ZNF367 & 23.837 & 337.056 & 3.822 & Up & $5.60 \mathrm{E}-08$ & $5.45 \mathrm{E}-06$ \\
\hline hsa_circ_0008883 & CAPN15 & 11.919 & 157.293 & 3.722 & Up & 0.000222228 & 0.005249134 \\
\hline hsa_circ_0005585 & NNT & 476.741 & 44.941 & -3.407 & Down & $1.74 \mathrm{E}-06$ & 0.000102458 \\
\hline hsa_circ_0017077 & LYST & 435.026 & 44.941 & -3.275 & Down & $7.63 \mathrm{E}-06$ & 0.000353697 \\
\hline hsa_circ_0001541 & ANKHD1 & 417.148 & 44.941 & -3.214 & Down & $1.43 \mathrm{E}-05$ & 0.000631106 \\
\hline hsa_circ_0001613 & SENP6 & 613.804 & 67.411 & -3.187 & Down & $9.68 \mathrm{E}-08$ & $8.75 E-06$ \\
\hline hsa_circ_0001346 & RNF13 & 1501.734 & 179.763 & -3.062 & Down & $5.76 \mathrm{E}-17$ & $2.48 \mathrm{E}-14$ \\
\hline hsa_circ_0002394 & SNX27 & 309.882 & 44.941 & -2.786 & Down & 0.000577398 & 0.012403449 \\
\hline hsa_circ_0046999 & CEP192 & 280.085 & 44.941 & -2.64 & Down & 0.00156603 & 0.024002196 \\
\hline hsa_circ_0000099 & $\mathrm{AMY} 2 \mathrm{~B}$ & 280.085 & 44.941 & -2.64 & Down & 0.00156603 & 0.023979743 \\
\hline hsa_circ_0001432 & MANBA & 965.401 & 157.293 & -2.618 & Down & $6.75 E-10$ & $8.84 \mathrm{E}-08$ \\
\hline hsa_circ_0001508 & $\mathrm{MSH} 3$ & 274.126 & 44.941 & -2.609 & Down & 0.001908118 & 0.028164097 \\
\hline
\end{tabular}


DECs in an OC group vs. OC + AL group comparison We also identified the circRNAs that were differentially expressed in the $\mathrm{OC}$ and $\mathrm{OC}+\mathrm{AL}$ groups. Figure 4a shows that the number of upregulated DECs (1434) was higher than the number of downregulated DECs (219) in this OC group vs. OPCS group comparison. As with the previous GO analysis, the functions of these DECs could be largely separated into the three categories cellular component, molecular function, and biological process (Fig. 4b). The most enriched items in the cellular component ("cell," "cell part," and "organelle"), molecular function ("binding"), and biological process ("cellular process" and "single-organism process") categories were respectively identical to those in the OPCS group vs. OC group comparison. The heat map in Fig. 4c shows the cluster relationships among these DECs (Fig. 4c). As with the OPCS group vs. OC group comparison, KEGG analysis of the top 20 DECs showed that enrichment was mostly in metabolic pathways and in the MAPK signaling pathway (Fig. 4d and Table 4).

\section{Network of circRNA-miRNA-mRNA}

To further examine the function of DECs, we screened the overlap of DECs among the three treatment groups: 110 circRNAs were differentially expressed among these groups.

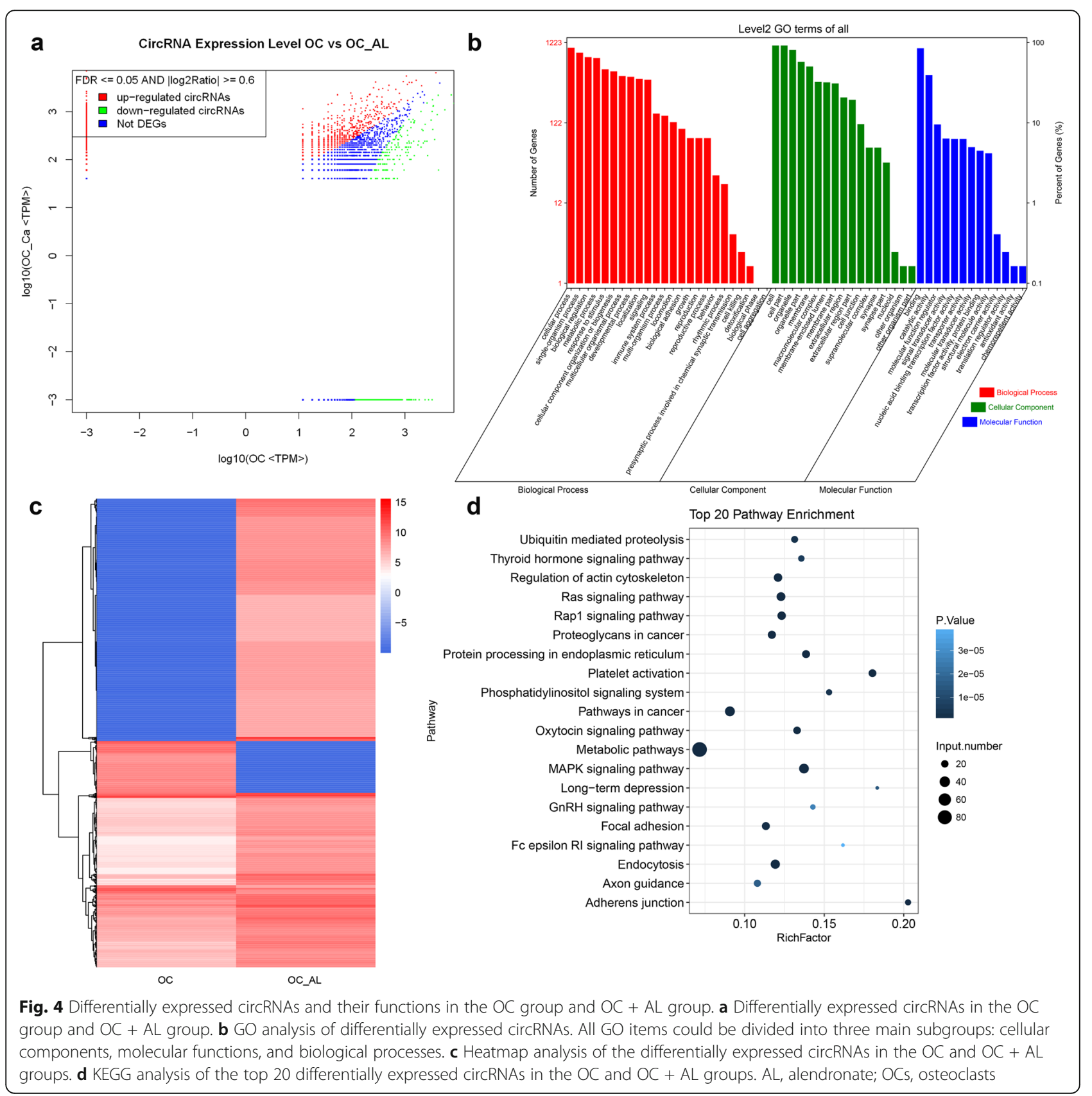


Table 4 Top 20 differential expression circRNA list between OC group and OC + AL group

\begin{tabular}{|c|c|c|c|c|c|c|c|}
\hline CircBase_ID & Gene ID & OC-TPM & OC_AL-TPM & $\log _{2}$ ratio (OC_AL/OC) & Up-downregulation & $\boldsymbol{P}$ value & FDR \\
\hline hsa_circ_0033144 & BCL11B & 11.919 & 2248.509 & 7.56 & Up & $9.34 \mathrm{E}-43$ & $1.14 \mathrm{E}-39$ \\
\hline hsa_circ_0009125 & HABP4 & 35.756 & 2981.719 & 6.382 & Up & $1.92 \mathrm{E}-52$ & $3.05 E-49$ \\
\hline hsa_circ_0083766 & EPHX2 & 11.919 & 830.971 & 6.123 & Up & $1.37 E-15$ & $2.14 \mathrm{E}-13$ \\
\hline hsa_circ_0007426 & FNBP1 & 11.919 & 782.09 & 6.036 & Up & $1.13 \mathrm{E}-14$ & $1.53 \mathrm{E}-12$ \\
\hline hsa_circ_0005035 & IGF1R & 17.878 & 977.613 & 5.773 & Up & $1.67 \mathrm{E}-17$ & $3.15 E-15$ \\
\hline hsa_circ_0063809 & CELSR1 & 17.878 & 830.971 & 5.539 & Up & $8.57 E-15$ & $1.19 \mathrm{E}-12$ \\
\hline hsa_circ_0005660 & NFIX & 11.919 & 488.806 & 5.358 & Up & $3.16 \mathrm{E}-09$ & $1.94 \mathrm{E}-07$ \\
\hline hsa_circ_0033476 & MARK3 & 11.919 & 488.806 & 5.358 & Up & $3.16 \mathrm{E}-09$ & $1.93 \mathrm{E}-07$ \\
\hline hsa_circ_0003489 & CDK8 & 11.919 & 488.806 & 5.358 & Up & $3.16 \mathrm{E}-09$ & $1.92 \mathrm{E}-07$ \\
\hline hsa_circ_0001730 & EPHB4 & 53.633 & 2150.748 & 5.326 & Up & $1.63 \mathrm{E}-34$ & $1.23 \mathrm{E}-31$ \\
\hline hsa_circ_0000566 & VRK1 & 1853.331 & 97.761 & -4.245 & Down & $3.16 \mathrm{E}-13$ & $3.86 \mathrm{E}-11$ \\
\hline hsa_circ_0000826 & ANKRD12 & 3629.191 & 244.403 & -3.892 & Down & $7.83 E-24$ & $2.53 E-21$ \\
\hline hsa_circ_0005615 & NFATC3 & 3259.717 & 244.403 & -3.737 & Down & $5.80 \mathrm{E}-21$ & $1.59 E-18$ \\
\hline hsa_circ_0004658 & EMILIN2 & 1251.445 & 97.761 & -3.678 & Down & $1.66 \mathrm{E}-08$ & $8.44 \mathrm{E}-07$ \\
\hline hsa_circ_0000284 & HIPK3 & 23306.676 & 1906.345 & -3.612 & Down & $1.52 E-139$ & $6.05 E-136$ \\
\hline hsa_circ_0086735 & UBAP2 & 2407.542 & 244.403 & -3.3 & Down & $1.85 E-14$ & $2.44 \mathrm{E}-12$ \\
\hline hsa_circ_0000994 & SLC8A1 & 7472.915 & 879.851 & -3.086 & Down & $2.15 \mathrm{E}-40$ & $2.13 E-37$ \\
\hline hsa_circ_0002538 & KLHL8 & 709.152 & 97.761 & -2.859 & Down & 0.000201768 & 0.003278317 \\
\hline hsa_circ_0006156 & FNDC3B & 4058.258 & 635.448 & -2.675 & Down & $7.46 \mathrm{E}-20$ & $1.77 \mathrm{E}-17$ \\
\hline hsa_circ_0006595 & ST3GAL3 & 1489.816 & 244.403 & -2.608 & Down & $9.13 \mathrm{E}-08$ & $3.79 E-06$ \\
\hline
\end{tabular}

Of the 110 DECs, 95 and 15 were upregulated and downregulated after $\mathrm{AL}$ treatment, respectively (Fig. 5a, b). In addition, we constructed a circRNA-miRNA-mRNA network. The circRNAs related to OC differentiation were selected using the following criteria: (1) circRNAs were sourced from exons, and (2) the length of circRNAs ranged from 300 to $1200 \mathrm{bp}$. Figure 5 shows that hsa_circ_0002922 bound to hsa-miR-181b-5p to regulate the expression of MAP2K1 and hsa_circ_0007710 bound to hsa-miR-197-3p to regulate the expression of MAPK1, and it also bound to hsa-miR-20a-5p to regulate the expression of MAPK9. The 20 top DECs are shown in Table 5 . Figure $5 \mathrm{c}$ shows that
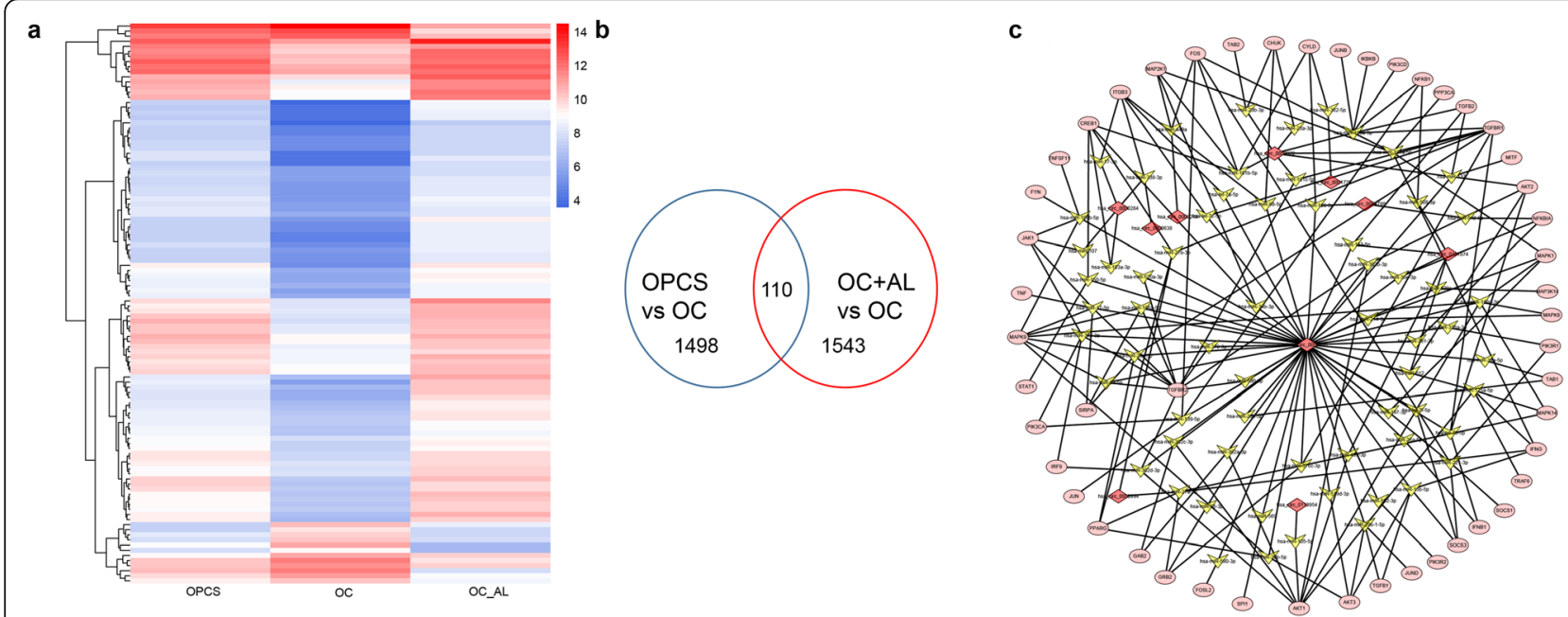

Fig. 5 Differentially expressed circRNAs and their regulation networks using the top 20 differentially expressed circRNAs in the OC, OPCS, and OC + AL groups. a Heatmap analysis of the differentially expressed circRNAs among the three treatment groups. $\mathbf{b}$ Venn diagram showing the relationships between the differentially expressed circRNAs among the three treatment groups. c circRNA-miRNA-mRNA regulation network analysis of the top 20 differentially expressed circRNAs among the three treatment groups 


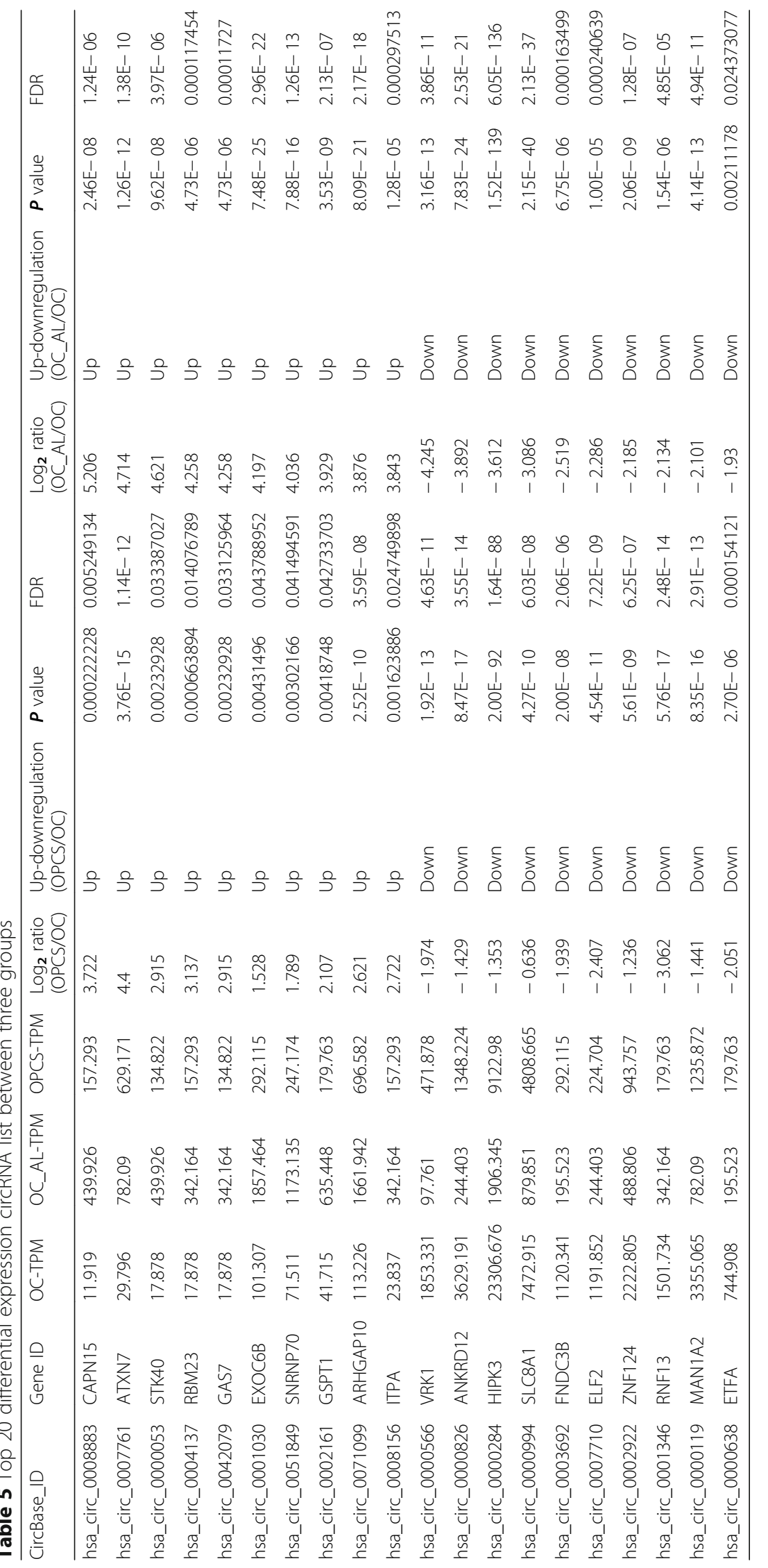


the top 10 DECs played key roles in the circRNA-miRNAmRNA network. Furthermore, the expression levels of 10 DECs in the key networks (hsa_circ_0000284, hsa_circ 0000638, hsa_circ_0000994, hsa_circ_0001776, hsa_circ 0002922, hsa_circ_0003249, hsa_circ_0007710, hsa_circ 0094789, hsa_circ_0113954, and hsa_circ_0101874) were also validated in the three treatment groups using $\mathrm{qPCR}$ (Fig. 6). The expression levels of hsa_circ_0000284, hsa circ_0000638, hsa_circ_0000994, hsa_circ_0001776, hsa circ_0002922, hsa_circ_0007710, and hsa_circ_0113954 were significantly increased in the $\mathrm{OC}$ group compared with those in the OPCS group $(P<0.05)$. However, the expression levels of these DECs were significantly decreased in the $\mathrm{OC}+\mathrm{AL}$ group compared with those in the $\mathrm{OC}$ group $(P<0.05)$. Furthermore, the expression levels of hsa circ_0003249, hsa_circ_0094789, and hsa_circ_0101874 were significantly decreased in the $\mathrm{OC}$ group compared with those in the OPCS group $(P<0.05)$. In contrast, the expression levels of these DECs were significantly increased in the $\mathrm{OC}+\mathrm{AL}$ group compared with those in the $\mathrm{OC}$ group $(P<0.05)$. Finally, we confirmed the identity of the top 10 DECs via agarose gel electrophoresis (Fig. 6).

\section{Discussion}

OCs are the main functional cells involved in bone resorption during bone remodeling. Their differentiation and activity are regulated by a variety of hormones and cytokines, including MCSF and RANKL: these two cytokines are critical for the regulation of OC-like cell differentiation in vitro [20]. Therefore, we selected both MCSF and RANKL to induce THP-1 cells into OCs and showed that they were functionally able to induce OC differentiation, which is a finding consistent with that of a previous study [21].

The anti-bone resorption effects of AL are mainly achieved via the inhibition of OCs. AL binds to nonhydrolyzable ATP analogs on the surface of OC membranes and inhibits the action of ATP-dependent intracellular enzymes, which in turn significantly inhibits the effects of OCs [22]; specifically, OCs lose the ability to absorb bone, which affects bone resorption and bone turnover rate $[23,24]$. Bisphosphonates such as AL are currently the most commonly used drugs for the treatment of OP; they can specifically bind to hydroxyapatite in the bone so are strong inhibitors of bone resorption but have little effect on other tissues [25]. Srisubut et al. found that $\mathrm{AL}$ promotes bone formation in bioactive glass grafting in rats [26], and Jensen et al. demonstrated that orally administering AL to dogs with prosthesis implantations promotes bone formation around a good prosthesis [27]. These studies indicate that AL plays a role in promoting local bone formation; similarly, we found that AL effectively inhibits OC differentiation.

We also found that 1394 and 214 circRNAs were differentially upregulated and downregulated, respectively, in the OPCS and OC groups, whereas 1434 and 219 circRNAs were differentially upregulated and downregulated, respectively, in the $\mathrm{OC}$ and $\mathrm{OC}+\mathrm{AL}$ groups. Notably, GO and KEGG analysis revealed that gene functions and signaling pathways were similarly enriched between these two groups of DECs. This indicates that the same genes might participate in both OC

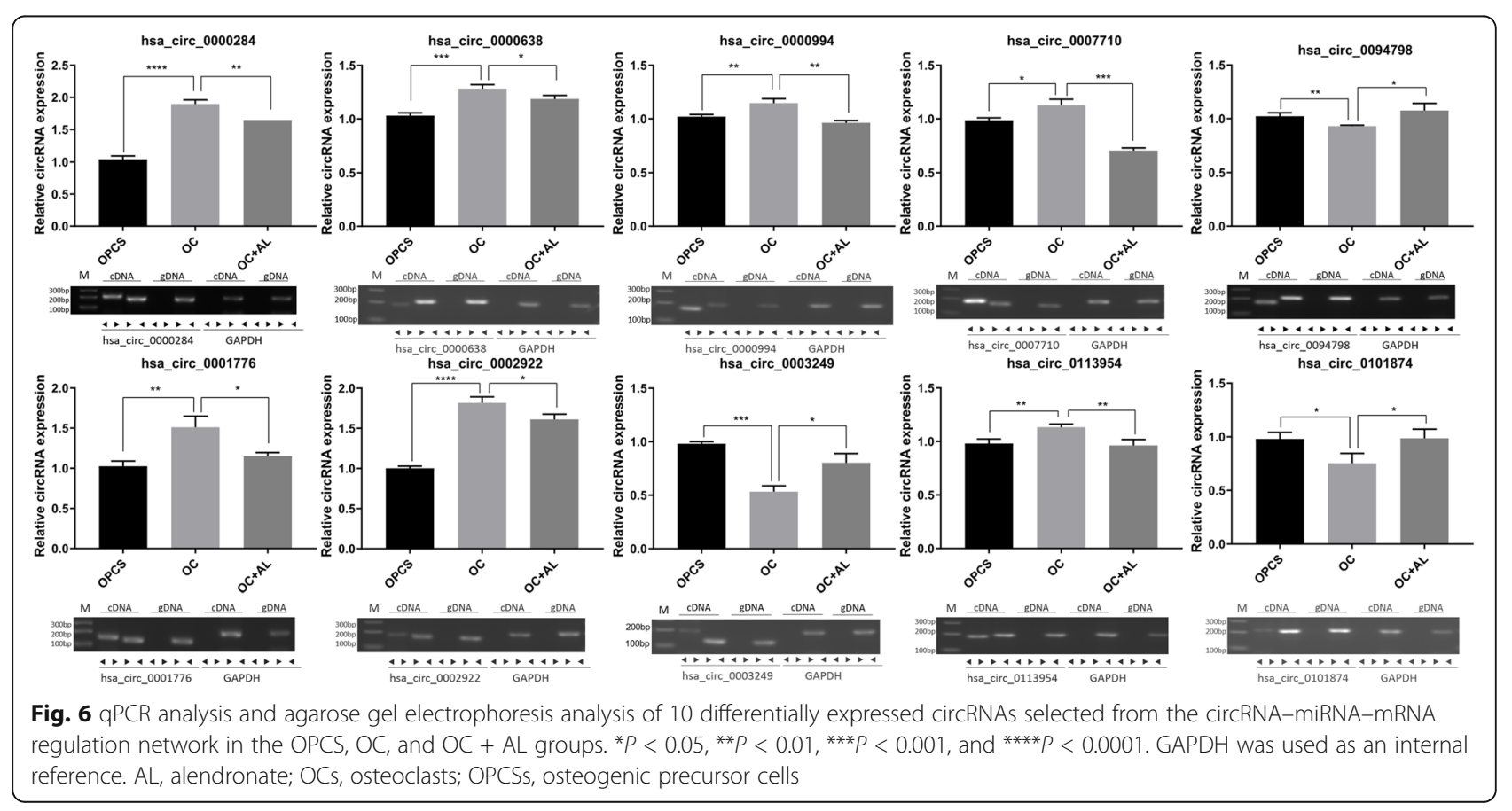


differentiation and inhibition. For example, the MAPK signaling pathway was enriched in both sets of DECs. The MAPK pathway is important in the signaling network of eukaryotic cells: it transduces extracellular mitosis signals to the cell nuclei [28]. Activated MAPK regulates a variety of cellular physiological processes such as growth, division, differentiation, and apoptosis through phosphorylation and ubiquitination [29]. Previous studies have shown that the MAPK pathway regulates the osteoprotegerin/RANKL intracellular pathway involved in the balance of bone metabolism.

The MAPKs associated with OC differentiation mainly comprise three subfamilies: extracellular signal-regulated protein kinase (ERK), p38MAPK, and c-Jun aminoterminal kinase [30]. The MAPK pathway is also considered to be an important pathway in inflammatory bone injury [31]. Other studies have shown that the p38 inhibitor SB203580 and the ERK inhibitor U0126 significantly reduce the activity of TRAP during OC formation and thereby inhibit the formation and function of OCs [32]. In addition, the circRNA CDR1as can inhibit the expression of miR-7, which releases the negative regulation of miR-7 on growth differentiation factor 5 , further activates the p38MAPK pathway and $\mathrm{pSmad} 1 / 5 / 8$, and promotes osteogenesis of periodontal ligament stem cells [33]. These results are consistent with our finding that circRNAs might bind to miRNA to regulate the MAPK pathway during differentiation of OCs treated with AL.

To date, there is a lack of research on circRNAs in the skeletal system. Some early studies have reported the expression of long non-coding RNAs during chondrocyte differentiation and osteoblast differentiation [34]; however, the expression and function of circRNAs in the process of OC formation had previously not been demonstrated. Thus, our study is the first to report the expression profiles of circRNAs during the differentiation of OCs treated with AL.

\section{Conclusions}

In summary, here, we successfully induced OPCSs to differentiate into OCs, showed that AL could suppress OPCSs from differentiating into OCs, and identified 10 DECs that were involved in the regulation of this process. These 10 circRNAs might be important to the treatment of OP; this speculation requires further enquiry.

\footnotetext{
Abbreviations

OP: Osteoporosis; OC: Osteoclast; circRNAs: Circular RNAs; AL: Alendronate; IL: Interleukin; MCSF: Macrophage colony-stimulating factor; RANKL: Receptor activator of nuclear factor-KB ligand; PMA: Phorbol-12 myristate-13 acetate; OPCS: Osteoclast precursor; TRAP: Tartrate-resistant acid phosphatase; CTSK: Cathepsin K; DEC: Differentially expressed circRNA; MAPK: Mitogenactivated protein kinase; ERK: Extracellular signal-regulated protein kinase
}

\section{Authors' contributions}

Guofeng Huang and Zhengi Ding conceived and designed the study and critically revised the manuscript. Jianbiao Lin, Shaofeng Ma, and Cong Zhu performed the experiments, analyzed the data, and drafted the manuscript. Changqing Chen, Weibin Lin, and Canbin Lin participated in study design, study implementation, and manuscript revision. All authors read and approved the final manuscript.

\section{Funding}

This work was supported by the Natural Science Foundation of Fujian Province of China [grant number 2016 J01423], Military Youth Training Program of China [grant number 19QNP046], Scientific Research Projects on Military Logistics [grant number CNJ16C013], and Youth Training Program of 18th Sub-region.

\section{Availability of data and materials}

Any information used and analyzed during this study is available from the corresponding author on reasonable request.

Ethics approval and consent to participate

Not applicable.

\section{Consent for publication}

Not applicable.

\section{Competing interests}

The authors declare that they have no competing interests.

\section{Author details}

${ }^{1}$ Center for Orthopedics, Affiliated Southeast Hospital of Xiamen University/ 909th Hospital of People's Liberation Army, 269 Zhanghua Middle Road, Zhangzhou 363000, Fujian, China. 'Obstetrics and Gynecology Department, Affiliated Southeast Hospital of Xiamen University/909th Hospital of People's Liberation Army, Zhangzhou, China.

Received: 6 November 2019 Accepted: 20 May 2020

Published online: 01 July 2020

\section{References}

1. Osterhoff G, Morgan EF, Shefelbine SJ, Karim L, McNamara LM, Augat P. Bone mechanical properties and changes with osteoporosis. Injury. 2016; 47(Suppl 2):S11-20.

2. Tella SH, Gallagher JC. Prevention and treatment of postmenopausal osteoporosis. J Steroid Biochem Mol Biol. 2014;142:155-70.

3. Faienza MF, Ventura A, Marzano F, Cavallo L. Postmenopausal osteoporosis: the role of immune system cells. Clin Dev Immunol. 2013;2013:575936.

4. Tanaka S, Nakamura K, Takahasi N, Suda T. Role of RANKL in physiological and pathological bone resorption and therapeutics targeting the RANKLRANK signaling system. Immunol Rev. 2005;208:30-49.

5. Matsuoka K, Park KA, Ito M, Ikeda K, Takeshita S. Osteoclast-derived complement component 3a stimulates osteoblast differentiation. J Bone Miner Res. 2014;29(7):1522-30.

6. Zaidi M. Skeletal remodeling in health and disease. Nat Med. 2007;13(7): 791-801.

7. Iwamoto J, Takeda T, Sato Y. Effects of antifracture drugs in postmenopausal, male and glucocorticoid-induced osteoporosis--usefulness of alendronate and risedronate. Expert Opin Pharmacother. 2007;8(16):2743-56.

8. Iwamoto J, Sato Y, Takeda T, Matsumoto H. Hip fracture protection by alendronate treatment in postmenopausal women with osteoporosis: a review of the literature. Clin Interv Aging. 2008;3(3):483-9.

9. Radicioni M, Cremonesi G, Baraldi E, Leuratti C, Mariotti F. Pharmacokinetic and tolerability of i.m. disodium clodronate $200 \mathrm{mg} /$ lidocaine $1 \%$, given twice monthly, in comparison with i.m. disodium clodronate $100 \mathrm{mg} /$ lidocaine $1 \%$, given weekly, in healthy postmenopausal female patients. Int J Clin Pharmacol Ther. 2013;51(4):313-22.

10. Patop IL, Wust S, Kadener S. Past, present, and future of circRNAs. 2019; 38(16):e100836.

11. Wang Y, Zhang J, Li J, Gui R, Nie X, Huang R. CircRNA_014511 affects the radiosensitivity of bone marrow mesenchymal stem cells by binding to miR-29b-2-5p. Bosn J Basic Med Sci. 2019;19(2):155-63. 
12. Zhou ZB, Huang GX, Fu Q, Han B, Lu JJ, Chen AM, et al. circRNA.33186 contributes to the pathogenesis of osteoarthritis by sponging miR-127-5p. Mol Ther. 2019;27(3):531-41.

13. Chen X, Ouyang Z, Shen Y, Liu B, Zhang Q, Wan L, et al. CircRNA_ 28313/miR-195a/CSF1 axis modulates osteoclast differentiation to affect OVX-induced bone absorption in mice. RNA Biol. 2019;16(9): 1249-62.

14. Jia J, Feng $X, X u$ W, Yang S, Zhang Q, Liu X, et al. MiR-17-5p modulates osteoblastic differentiation and cell proliferation by targeting SMAD7 in non-traumatic osteonecrosis. Exp Mol Med. 2014;46:e107.

15. Li ZH, Si Y, Xu G, Chen XM, Xiong H, Lai L, et al. High-dose PMA with RANKL and MCSF induces THP1 cell differentiation into human functional osteoclasts in vitro. Mol Med Rep. 2017;16(6):8380-4.

16. Park EK, Jung HS, Yang HI, Yoo MC, Kim C, Kim KS. Optimized THP-1 differentiation is required for the detection of responses to weak stimuli. Inflamm Res. 2007:56(1):45-50.

17. Pasquier J, Thomas B, Hoarau-Vechot J, Odeh T, Robay A, Chidiac O, et al. Circulating microparticles in acute diabetic Charcot foot exhibit a high content of inflammatory cytokines, and support monocyte-to-osteoclast cell induction. Sci Rep. 2017;7(1):16450.

18. Takashiba S, Dyke TEV, Amar S, Murayama Y, Soskolne AW, Shapira L. Differentiation of monocytes to macrophages primes cells for lipopolysaccharide stimulation via accumulation of cytoplasmic nuclear factor KB. Infect Immun. 1999;67(11):5573-8.

19. Oshima K, Nampei A, Matsuda M, Iwaki M, Fukuhara A, Hashimoto J, et al. Adiponectin increases bone mass by suppressing osteoclast and activating osteoblast. 331(2):526.

20. Theoleyre S, Wittrant Y, Tat SK, Fortun Y, Redini F, Heymann D. The molecular triad OPG/RANK/RANKL: involvement in the orchestration of pathophysiological bone remodeling. Cytokine Growth Factor Rev. 2004; 15(6):457-75.

21. Zur Y, Rosenfeld L, Keshelman CA, Dalal N, Guterman-Ram G, Orenbuch A, et al. A dual-specific macrophage colony-stimulating factor antagonist of cFMS and alphavbeta3 integrin for osteoporosis therapy. PLoS Biol. 2018; 16(8):e2002979.

22. Chavassieux PM, Arlot ME, Reda C, Wei L, Yates AJ, Meunier PJ. Histomorphometric assessment of the long-term effects of alendronate on bone quality and remodeling in patients with osteoporosis. J Clin Invest. 1997;100(6):1475-80

23. Saha H, Castren-Kortekangas P, Ojanen S, Juhakoski A, Tuominen J, Tokola $\mathrm{O}$, et al. Pharmacokinetics of clodronate in renal failure. J Bone Miner Res. 1994;9(12):1953-8.

24. Hooper MJ, Ebeling PR, Roberts AP, Graham JJ, Nicholson GC, D'Emden $M$, et al. Risedronate prevents bone loss in early postmenopausal women: a prospective randomized, placebo-controlled trial. Climacteric. 2005;8(3):251-62.

25. Kimmel DB. Mechanism of action, pharmacokinetic and pharmacodynamic profile, and clinical applications of nitrogen-containing bisphosphonates. J Dent Res. 2007:86(11):1022-33.

26. Srisubut S, Teerakapong A, Vattraphodes T, Taweechaisupapong S. Effect of local delivery of alendronate on bone formation in bioactive glass grafting in rats. Oral Surg Oral Med Oral Pathol Oral Radiol Endod. 2007;104(4):e11-6.

27. Jensen TB, Bechtold JE, Chen X, Søballe K. Systemic alendronate treatment improves fixation of press-fit implants: a canine study using nonloaded implants. J Orthop Res. 2007;25(6):772-8.

28. Cargnello M, Roux PP. Activation and function of the MAPKs and their substrates, the MAPK-activated protein kinases. Microbiol Mol Biol Rev. 2011; 75(1):50-83.

29. Zhang W. MAPK signal pathways in the regulation of cell proliferation in mammalian cells. Cell Research (English version). 2002.

30. Zhu ZA, Yu YP, Zheng MH, Dai KR, Jiang Q, Tang TT, et al. OA-4 inhibits osteoclast formation and bone resorption via suppressing RANKL induced P38 signaling pathway. Curr Med Chem. 2014;21(5):641-9.

31. Mine Y, Makihira S, Yamaguchi Y, Tanaka H, Nikawa H. Involvement of ERK and p38 MAPK pathways on Interleukin-33-induced RANKL expression in osteoblastic cells. Cell Biol Int. 2014;38(5):655-62.

32. Ma X, Liu Y, Zhang Y, Yu X, Wang W, Zhao D. Jolkinolide B inhibits RANKLinduced osteoclastogenesis by suppressing the activation NF-KB and MAPK signaling pathways. Biochem Biophys Res Commun. 2014;445(2):282-8.
33. Li X, Zheng Y, Zheng Y, Huang Y, Zhang Y, Jia L, et al. Circular RNA CDR1as regulates osteoblastic differentiation of periodontal ligament stem cells via the miR-7/GDF5/SMAD and p38 MAPK signaling pathway. Stem Cell Res Ther. 2018:9(1):232.

34. Wang L, Li Z, Li Z, Yu B, Wang Y. Long noncoding RNAs expression signatures in chondrogenic differentiation of human bone marrow mesenchymal stem cells. Biochem Biophys Res Commun. 2015;456(1):459-64.

\section{Publisher's Note}

Springer Nature remains neutral with regard to jurisdictional claims in published maps and institutional affiliations.
Ready to submit your research? Choose BMC and benefit from:

- fast, convenient online submission

- thorough peer review by experienced researchers in your field

- rapid publication on acceptance

- support for research data, including large and complex data types

- gold Open Access which fosters wider collaboration and increased citations

- maximum visibility for your research: over $100 \mathrm{M}$ website views per year

At BMC, research is always in progress.

Learn more biomedcentral.com/submissions 